\title{
No-dipole-hair theorem for higher-dimensional static black holes
}

\author{
Roberto Emparan \\ Institució Catalana de Recerca i Estudis Avançats (ICREA), Passeig Lluís Companys 23, E-08010 Barcelona, \\ Spain and Departament de Física Fonamental and Institut de Ciències del Cosmos, \\ Universitat de Barcelona, Martí i Franquès 1, E-08028 Barcelona, Spain \\ Seiju Ohashi and Tetsuya Shiromizu \\ Department of Physics, Kyoto University, Kyoto 606-8502, Japan
}

(Received 27 July 2010; published 20 October 2010)

\begin{abstract}
We prove that static black holes in $n$-dimensional asymptotically flat spacetime cannot support nontrivial electric $p$-form field strengths when $(n+1) / 2 \leq p \leq n-1$. This implies, in particular, that static black holes cannot possess dipole hair under these fields.
\end{abstract}

DOI: 10.1103/PhysRevD.82.084032

PACS numbers: 04.50.Gh, 04.70.Bw

\section{INTRODUCTION}

It has been known for several years that the properties of higher-dimensional black holes can differ significantly from the rigidly constrained character of four-dimensional black holes [1]. References [2,3] trace the origin of the new physics back to the possibility of having two parametrically different length scales along the horizon, which allows higher-dimensional black holes to exhibit black brane-like behavior that cannot occur in four dimensions. Typically, one of the scales is associated to the black hole mass and the other to its angular momentum, which in five or more dimensions can be arbitrarily large for a given mass. As the two scales begin to separate, new phenomena set in, such as black hole nonuniqueness and horizon instabilities.

This observation suggests that the distinctively higherdimensional features of black holes arise only at sufficiently large rotation. In particular, it leads us to expect that the properties of static black holes should be qualitatively very similar to those of four-dimensional black holes. There is already good evidence for this. Prompted by the discovery of black rings and the nonuniqueness of stationary black holes that they entail [4], Ref. [5] showed that asymptotically flat, static vacuum black holes are instead unique: the only solution is the SchwarzschildTangherlini spacetime. Afterward it was also shown that this solution is dynamically stable to linearized perturbations [6]. While we expect that uniqueness (for solutions with connected horizons) and stability are valid in a finite range of values of the angular momenta, the precise upper limits are still unknown in general (see [7,8] for some recent progress in this direction).

It is clearly of interest to study how these results are extended when gauge fields are present. A charge on the black hole introduces an independent length scale, namely, the charge radius. A separation of scales occurs as extremality is approached, but this occurs in directions transverse to the horizon instead of parallel to it, and is in fact an effect well-known in four dimensions too. So, again, the onset of qualitatively new higher-dimensional features seems to require a minimum amount of rotation. In this direction, Ref. [9] has proven the uniqueness of the $n$-dimensional static black holes electrically charged under a two-form field strength, and Ref. [10] has studied their stability.

A more distinctive property of gauge fields in higher dimensions is the possibility that a black hole couples electrically to $p$-form field strengths $H_{(p)}$ with $p>2$. An asymptotically flat black hole in $n$ dimensions cannot have a conserved monopolar electric charge under this field. One might expect that the integral of $* H_{(p)}$ over a sphere $S^{n-p}$ near asymptotically flat infinity gives a conserved charge. However, this is not the case when $p>2$, since the $S^{n-p}$ can be shrunk to a point in the $S^{n-2}$ in the asymptotically flat region and the integral vanishes. Nevertheless, the black hole can be the source of an electric dipole of this field. Indeed, Ref. [11] presented rotating black ring solutions with dipoles of a three-form field strength in five dimensions. Since the dipole is not a conserved charge, it is hair for the black hole. The generic existence of rotating black holes with dipoles of fields $H_{(p)}$ with $p \geq 3$ in dimensions $n \geq p+2$ is argued in [12].

Could a static black hole sport such dipole hairs? Intuitively, the dipole field can be regarded as sourced by a $(p-2)$-brane-like object extended along a compact $(p-2)$-cycle. This "brane" exerts a tension that, if the cycle is contractible, must be balanced by centrifugal rotation (this is indeed explicitly observed in the dipole rings of [11]). So this heuristic reasoning indicates that we should not expect a black hole to be able to support a dipole until it carries a sufficiently large angular momentum.

In this article we prove the impossibility of dipole hair for static black holes. The proof follows the one employed in the uniqueness theorem of higher-dimensional static black holes $[5,9,13,14]$. This type of proof was first developed by Bunting and Masood-ul-Alam in four dimensions [15]. Its extension to higher dimensions is quite nontrivial, since [15] used properties specific to four dimensions, but 
the approach was extended in $[5,13]$ in a manner that avoids the use of such properties.

Together with the gauge dipole, we will also consider the inclusion of scalar fields and scalar hair. Bekenstein proved that a static black hole cannot have scalar hair in four dimensions [16]. This no-hair theorem is easily extended to higher dimensions since the dimensionality does not enter into the proof. [16] However, this type of proof cannot be applied to systems where the scalar field couples to higher form fields.

The rest of this paper is organized as follows. In Sec. II, we prove the no-dipole-hair theorem in two steps: first we show that a static black hole cannot support nontrivial $p$-form fields when $(n+1) / 2 \leq p \leq n-1$, and then we prove the uniqueness of the Schwarzschild-Tangherlini solution. In Sec. III, we discuss the outlook of this work and, in particular, the restriction on the values of $p$ to which the theorem applies.

\section{NO-DIPOLE-HAIR THEOREM}

We consider $n$-dimensional asymptotically flat solutions of theories described by the class of Lagrangians

$$
\mathcal{L}=R-\frac{1}{2}(\partial \phi)^{2}-\frac{1}{p !} e^{-\alpha \phi} H_{(p)}^{2},
$$

where $R$ is the $n$-dimensional Ricci scalar, $\phi$ is a dilaton with coupling $\alpha$, and $H_{(p)}$ is the field strength of a $(p-1)$ form field potential $B_{(p-1)}$,

$$
H_{(p)}=d B_{(p-1)} .
$$

Since we are interested in asymptotically flat spacetimes, we take $p \leq n-1$. A form field with $p=n$ does not have any dynamical degree of freedom and behaves like a cosmological constant, which would prevent asymptotic flatness.

We only consider electric fields of $H_{(p)}$. Note that via electric-magnetic duality we can always trade a magnetic charge or dipole under $H_{(p)}$ for an electric one under $H_{(n-p)}{ }^{1}$ However, we do not consider the possibility of simultaneous presence of dipoles and monopole charges of electric and magnetic type, e.g., in $n=p+2$ one can have solutions with both magnetic monopole charge and electric dipole of $H_{(p)}$. Sometimes these involve an additional Chern-Simons term in the action, which however is inconsequential for our analysis involving only electric fields. The uniqueness of $U(1)^{2}$-symmetric black holes in some such theories in five dimensions has been discussed in [18].

The equations of motion for the theories (1) are

$$
\nabla^{2} \phi=-\frac{\alpha}{p !} e^{-\alpha \phi} H_{(p)}^{2}
$$

\footnotetext{
${ }^{1}$ Reference [17] purports to study black holes with both electric and magnetic monopole charges under $H_{(n-2)}$, but if $n>4$ this is impossible for the reasons given above.
}

$$
\nabla_{M}\left(e^{-\alpha \phi} H^{M N_{1} \cdots N_{p-1}}\right)=0
$$

and

$$
\begin{aligned}
R_{M N}= & \frac{1}{2} \nabla_{M} \phi \nabla_{N} \phi+\frac{1}{p !} e^{-\alpha \phi} \\
& \times\left(p H_{M}^{I_{1} \cdots I_{p-1}} H_{N I_{1} \cdots I_{p-1}}-\frac{p-1}{n-2} g_{M N} H_{(p)}^{2}\right),
\end{aligned}
$$

where $\nabla_{M}$ is the covariant derivative with respect to $g_{M N}$, and $M, N=0, \ldots n-1$.

The metric of a static spacetime can be written as

$$
d s^{2}=g_{M N} d x^{M} d x^{N}=-V^{2}\left(x^{i}\right) d t^{2}+g_{i j}\left(x^{k}\right) d x^{i} d x^{j},
$$

where $x^{i}$ are spatial coordinates on $x^{0}=t=$ const surfaces $\Sigma$. In these coordinates, the event horizon is located at $V=0$, i.e., the Killing horizon. The static ansatz for the $(p-1)$-form potential is of the form

$$
B_{(p-1)}=\varphi_{i_{1} \cdots i_{p-2}}\left(x^{k}\right) d t \wedge d x^{i_{1}} \wedge \cdots \wedge d x^{i_{p-2}} .
$$

Then the only nontrivial component of the field strength is $H_{0 i_{1} \cdots 1_{p-1}}$. The metric components and the potential do not depend on $t$.

We shall prove the following theorem:

No-dipole-hair theorem.-The only static, asymptotically flat black hole solution for the theories (1) with electric $p$-form field strength, with $(n+1) / 2 \leq p \leq$ $n-1$, is the Schwarzschild-Tangherlini solution.

From the Einstein equation we have

$$
\begin{aligned}
R_{i j}= & { }^{(n-1)} R_{i j}-\frac{1}{V} D_{i} D_{j} V \\
= & \frac{1}{2} D_{i} \phi D_{j} \phi+\frac{1}{(p-2) !} e^{-\alpha \phi}\left(H_{i}^{0 k_{1} \cdots k_{p-2}} H_{j 0 k_{1} \cdots k_{p-2}}\right. \\
& \left.-\frac{1}{n-2} g_{i j} H_{0 k_{1} \cdots k_{p-1}} H^{0 k_{1} \cdots k_{p-1}}\right)
\end{aligned}
$$

and

$$
R_{00}=V D^{2} V=\frac{n-p-1}{(n-2)(p-1) !} e^{-\alpha \phi} H_{0}^{i_{1} \cdots i_{p-1}} H_{0 i_{1} \cdots i_{p-1}},
$$

where $D_{i}$ is the covariant derivative with respect to $g_{i j}$. From these we derive

$$
{ }^{(n-1)} R=\frac{e^{-\alpha \phi}}{(p-1) ! V^{2}} H_{0}^{i_{1} \cdots i_{p-1}} H_{0 i_{1} \cdots i_{p-1}}+\frac{1}{2}(D \phi)^{2}
$$

and

$$
D^{2} V=\frac{n-p-1}{(n-2)(p-1) !} \frac{e^{-\alpha \phi}}{V} H_{0}^{i_{1} \cdots i_{p-1}} H_{0 i_{1} \cdots i_{p-1}} .
$$

From the equations for the form field we obtain

$$
D_{i}\left(e^{-\alpha \phi} H_{j_{1} \cdots j_{p-2} 0}^{i}\right)=\frac{D_{i} V}{V} e^{-\alpha \phi} H_{j_{1} \cdots j_{p-2} 0}^{i} .
$$


The asymptotic behavior of $V, g_{i j}$, and $H_{(p)}$ is

$$
\begin{gathered}
V=1-\frac{m}{r^{n-3}}+O\left(1 / r^{n-2}\right) \\
g_{i j}=\delta_{i j}\left(1+\frac{2}{n-3} \frac{m}{r^{n-3}}\right)+O\left(1 / r^{n-2}\right) \\
H_{0 i_{1} \cdots i_{p-1}}=O\left(1 / r^{n-p+1}\right) .
\end{gathered}
$$

Observe that the falloff of $H_{(p)}$ is the appropriate one for a dipole field, or higher multipole components. In our proof this decay rate could be relaxed to that of a monopole field, $O\left(1 / r^{n-p}\right)$. However, as we explained in the introduction, when $p>2$ electric monopole charges are incompatible with asymptotic flatness.

We also assume regularity on the event horizon. To this effect, we compute the curvature invariant

$$
\begin{aligned}
& R_{M N K L} R^{M N K L} \\
& ={ }^{(n-1)} R_{i j k l}{ }^{(n-1)} R^{i j k l}+4^{(n-1)} R_{0 i 0 j}{ }^{(n-1)} R^{0 i 0 j} \\
& ={ }^{(n-1)} R_{i j k l}{ }^{(n-1)} R^{i j k l}+4 \frac{D_{i} D_{j} V D^{i} D^{j} V}{V^{4}} \\
& ={ }^{(n-1)} R_{i j k l}{ }^{(n-1)} R^{i j k l}+\frac{4(n-2)}{(n-3) V^{2} \rho^{2}} \\
& \quad \times\left[k_{a b} k^{a b}+k^{2}+\mathcal{D}_{a} \rho \mathcal{D}^{a} \rho\right] .
\end{aligned}
$$

Here we have used the fact that the spatial metric can be written as

$$
g_{i j} d x^{i} d x^{j}=\rho^{2} d V^{2}+h_{a b} d x^{a} d x^{b},
$$

where $x^{a}$ is the coordinate on the level surfaces of $V . \mathcal{D}_{a}$ is the covariant derivative with respect to $h_{a b} . k_{a b}$ is the extrinsic curvature of $V=$ const surface and $\rho:=$ $\left|D^{i} V D_{i} V\right|^{-1 / 2}$. Then, from Eq. (16), one can easily see that

$$
\left.k_{a b}\right|_{V=0}=\left.\mathcal{D}_{a} \rho\right|_{V=0}=0
$$

hold on the event horizon. From the Einstein equation, we can also easily see that regularity implies $H_{0 i_{1} \cdots i_{p-1}}=0$ on the event horizon; see Eq. (10).

Let us consider the conformal transformation defined by

$$
\tilde{g}_{i j}=\Omega_{ \pm}^{2} g_{i j},
$$

where

$$
\Omega_{ \pm}=\left(\frac{1 \pm V}{2}\right)^{2 /(n-3)}=: \omega_{ \pm}^{2 /(n-3)} .
$$

This conformal transformation is the same as the one employed in the proof for the vacuum case [5,13]. Now we have two manifolds, $\left(\tilde{\Sigma}^{+}, \tilde{g}^{+}\right)$and $\left(\tilde{\Sigma}^{-}, \tilde{g}^{-}\right)$. The Ricci scalar of $\tilde{\Sigma}^{ \pm}$is

$$
\begin{aligned}
\Omega_{ \pm}^{2}{ }^{(n-1)} \tilde{R}_{ \pm}= & { }^{(n-1)} R-2(n-2) D^{2} \ln \Omega_{ \pm} \\
& -(n-3)(n-2)\left(D \ln \Omega_{ \pm}\right)^{2} \\
= & { }^{(n-1)} R \mp \frac{2(n-2)}{n-3} \omega_{ \pm}^{-1} D^{2} V \\
= & \frac{1}{(p-1) !} \frac{e^{-\alpha \phi}}{V^{2}} \frac{\lambda_{ \pm}}{\omega_{ \pm}} H_{0}^{i_{1} \cdots i_{p-1}} H_{0 i_{1} \cdots i_{p-1}} \\
& +\frac{1}{2}(D \phi)^{2},
\end{aligned}
$$

where

$$
\lambda_{ \pm}:=\frac{1 \mp \frac{3 n-4 p-1}{n-3} V}{2} .
$$

Since $0 \leq V \leq 1$, the $\lambda_{ \pm}$are positive-definite if

$$
\frac{n+1}{2} \leq p \leq n-1 \text {. }
$$

Under this condition the positivity of ${ }^{(n-1)} \tilde{R}_{ \pm}$follows. We will use this result later.

On $\tilde{\Sigma}^{+}$the asymptotic behavior of the metric becomes

$$
\tilde{g}_{i j}^{+}=\left(1+O\left(1 / r^{n-2}\right)\right) \delta_{i j}
$$

and therefore the Arnowitt-Deser-Misner (ADM) mass vanishes there. On $\tilde{\Sigma}^{-}$, the metric behaves like

$$
\begin{aligned}
\tilde{g}_{i j}^{-} d x^{i} d x^{j} & =\frac{(m / 2)^{4 /(n-3)}}{r^{4}} \delta_{i j} d x^{i} d x^{j}+O\left(1 / r^{5}\right) \\
& =(m / 2)^{4 /(n-3)}\left(d \rho^{2}+\rho^{2} d \Omega_{n-2}^{2}\right)+O\left(\rho^{5}\right),
\end{aligned}
$$

where we set $\rho:=1 / r$. From this, we see that infinity on $\Sigma$ corresponds to a point, which we denote as $q$.

Let us construct a new manifold $\left(\tilde{\Sigma}, \tilde{g}_{i j}\right):=\left(\tilde{\Sigma}^{+}, \tilde{g}_{i j}^{+}\right) \cup$ $\left(\tilde{\Sigma}^{-}, \tilde{g}_{i j}^{-}\right) \cup\{q\}$ by gluing the two manifolds $\left(\tilde{\Sigma}^{+}, \tilde{g}_{i j}^{+}\right)$and $\left(\tilde{\Sigma}^{-}, \tilde{g}_{i j}^{-}\right)$along the surface $V=0$ and adding the point $q .^{2}$

The calculations above imply that $\left(\tilde{\Sigma}, \tilde{g}_{i j}\right)$ has zero mass and non-negative Ricci scalar. Note also that near the point $q$ (which corresponds to $r \rightarrow \infty$ ) we have ${ }^{(n-1)} \tilde{R}_{-}=$ $O\left(r^{-(n-3)}\right)$, so $\tilde{\Sigma}^{-}$is regular at $q .^{3}$ Thus $\tilde{\Sigma}$ is a Riemannian manifold with non-negative Ricci scalar and zero ADM mass. Then, by the positive energy theorem [19], $\tilde{\Sigma}$ is flat. So the metric $\tilde{g}_{i j}$ is flat and

$$
H_{0 i_{1} \cdots i_{p-1}}=0 \text { and } \phi=\text { const }
$$

\footnotetext{
${ }^{2}$ Note that the resulting manifold $\tilde{\Sigma}$ is $C^{1}$ on the surface $V=$ 0 . This is as in the vacuum case [5], since the conformal transformation is the same, as mentioned above.

${ }^{3}$ Even if the system has monopole charge, ${ }^{(n-1)} \tilde{R}_{-}=$ $O\left(r^{-(n-5)}\right)$. So $\tilde{\Sigma}^{-}$will be regular whenever $n \geq 5$. Our method of proof does not depend on which hair the system has. If $H_{0 i_{1} \cdots i_{p-1}}=O\left(1 / r^{s}\right),{ }^{(n-1)} \tilde{R}_{-}=O\left(r^{n-2 p-2 s+5}\right)$. Then regularity requires $s \geq(n+1) / 2-(p-2)$.
} 
hold. ${ }^{4}$ That is, asymptotically flat static black holes in $n$ dimensions cannot support an electric dipole $p$-form field strength with $p$ in the range (23), nor a nontrivial scalar field.

Once we have ruled out the possibility of nontrivial $p$-form and scalar fields, the problem is exactly the same as in vacuum and the results of [5] imply the uniqueness of the Schwarzschild-Tangherlini solution. For the sake of completeness, we briefly review this argument.

We have seen that $\tilde{\Sigma}^{+}$must be flat space. In addition, we can check that the extrinsic curvature of the surface $V=0$ on $\tilde{\Sigma}^{+}$is proportional to its induced metric with a constant coefficient. According to Kobayashi and Nomizu [21], such a surface in flat space is spherically symmetric. Next, we define the function $v$ by

$$
v=\frac{2}{1+V} \text {. }
$$

It is easy to see that it is a harmonic function on flat space $\tilde{\Sigma}^{+}$, that is

$$
\partial^{2} v=0 .
$$

The boundary corresponding to the horizon is spherically symmetric. So the problem is reduced to the familiar one of an electrostatic potential with spherical boundary in flat space. We can easily see that the level surfaces of $v$ are spherically symmetric in the full region of $\tilde{\Sigma}^{+}$. So we have shown that $\Sigma$ is spherically symmetric and then the spacetime must be the Schwarzschild-Tangherlini spacetime. This completes our proof.

\section{OUTLOOK}

We have proven a no-dipole-hair theorem for $p$-form fields with $p$ in the range (23). The proof can be straightforwardly extended to theories containing several electric form fields $H_{\left(p_{i}\right)}$ of different rank $p_{i}$, each with its own coupling $\alpha_{i}$ to the dilaton, as long as each of the $p_{i}$ satisfies (23).
As mentioned above, the upper bound on $p$ is a natural one given the requirement of asymptotic flatness. But the physical motivation for the lower bound, if any, is unclear. Could static black holes support dipoles when $p<(n+$ $1) / 2$ ? The answer when $p=2$ is known: the uniqueness theorem of [9] affirms that a static black hole can have electric monopole charge, but not any higher multipole. However, here we are more interested in $p>2$ where monopoles are not allowed. For instance, could there be static black holes in $n \geq 6$ with electric three-form, i.e., string, dipole? The heuristic argument presented in the introduction would seem to run counter to this possibility, but maybe this argument misses a way to balance or cancel the tension of dipole sources that does not involve centrifugal forces. If this were the case it would be a striking new feature of static black holes afforded by higher dimensions. Alternatively, and more simply, maybe our nodipole-hair theorem can be strengthened to rule out all $p$-form dipoles whenever $p \leq n-1$. This issue seems worthy of further investigation.

\section{ACKNOWLEDGMENTS}

This work was initiated at Yukawa Institute, Kyoto University. We thank Misao Sasaki, Norihiro Tanahashi, and Takahiro Tanaka for useful comments. R.E. also thanks KIAS (Seoul) for hospitality while part of this work was in progress. S.O. thanks Professor Takashi Nakamura for his continuous encouragement. This work was supported by the Grant-in-Aid for the Global COE Program from the Ministry of Education, Culture, Sports, Science, and Technology (MEXT) of Japan. R.E. is partially supported by DURSI 2009 SGR 168, MEC FPA 2007-66665-C02 and CPAN CSD2007-00042 ConsoliderIngenio 2010. T. S. is partially supported by the Ministry of Education, Science, Sports, and Culture of Japan, Grant-inAid for Scientific Research Nos. 21244033, 21111006, 20540258, and 19GS0219, the Japan-U.K. Research Cooperative Programs.
[1] R. Emparan and H. S. Reall, Living Rev. Relativity 11, 6 (2008).

[2] R. Emparan and R. C. Myers, J. High Energy Phys. 09 (2003) 025.

[3] R. Emparan, T. Harmark, V. Niarchos, and N. A. Obers, Phys. Rev. Lett. 102, 191301 (2009); J. High Energy Phys. 03 (2010) 63.

\footnotetext{
${ }^{4}$ More precisely, this argument requires that the surface $t=$ const is a spin-manifold or a manifold of dimension less than eight [20].
}

[4] R. Emparan and H. S. Reall, Phys. Rev. Lett. 88, 101101 (2002).

[5] G. W. Gibbons, D. Ida, and T. Shiromizu, Prog. Theor. Phys. Suppl. 148, 284 (2002).

[6] A. Ishibashi and H. Kodama, Prog. Theor. Phys. 110, 901 (2003).

[7] O. J. C. Dias, P. Figueras, R. Monteiro, J. E. Santos, and R. Emparan, Phys. Rev. D 80, 111701 (2009).

[8] M. Shibata and H. Yoshino, Phys. Rev. D 81, 021501 (2010).

[9] G. W. Gibbons, D. Ida, and T. Shiromizu, Phys. Rev. Lett. 89, 041101 (2002); Phys. Rev. D 66, 044010 (2002). 
[10] H. Kodama and A. Ishibashi, Prog. Theor. Phys. 111, 29 (2004).

[11] R. Emparan, J. High Energy Phys. 03 (2004) 064.

[12] R. Emparan, T. Harmark, V. Niarchos, and N. A. Obers (unpublished).

[13] S. Hwang, Geometriae Dedicata 71, 5 (1998).

[14] M. Rogatko, Phys. Rev. D 67, 084025 (2003).

[15] G. L. Bunting and A. K.M. Masood-ul-Alam, Gen. Relativ. Gravit. 19, 147 (1987).

[16] J. D. Bekenstein, Phys. Rev. D 5, 1239 (1972).

[17] M. Rogatko, Phys. Rev. D 70, 044023 (2004).
[18] S. Hollands and S. Yazadjiev, Classical Quantum Gravity 25, 095010 (2008); S. Tomizawa, Y. Yasui, and A. Ishibashi, Phys. Rev. D 79, 124023 (2009); 81, 084037 (2010); J. Armas and T. Harmark, J. High Energy Phys. 05 (2010) 93.

[19] E. Witten, Commun. Math. Phys. 80, 381 (1981).

[20] R. Schoen and S. T. Yau, Commun. Math. Phys. 65, 45 (1979); R. Schoen, in Topics in Calculus of Variations (Montecatini Terme, 1987), Lecture Notes in Mathematics Vol. 1365 (Springer, New York, 1989).

[21] S. Kobayashi and K. Nomizu, Foundations of Differential Geometry (Interscience, New York, 1969), Vol. II, Sec. VII, Theorem 5.1. 